\title{
Speech intelligibility reduces over distance from an attended location: Evidence for an auditory spatial gradient of attention
}

\author{
Kachina Allen, David Alais, and Simon Carlile \\ University of Sydney, Sydney, New South Wales, Australia
}

\begin{abstract}
Speech reception thresholds (SRTs) were measured at a central focus of attention and at $20^{\circ}, 40^{\circ}$, and $60^{\circ}$ locations distant in azimuth. Measurements were taken with one target collocated with two maskers, or with maskers flanking the target by $\pm 20^{\circ}$. For $80 \%$ of trials, the target was played from the attended location ("expected"), and $20 \%$ came from another ("unexpected") location. For collocated stimuli, SRTs worsened with increasing distance from the expected location by $2.1 \mathrm{~dB}$ over the $60^{\circ}$ azimuth and by $5.1 \mathrm{~dB}$ for spatially separated target and maskers. In spatially separated conditions, a $2.9-\mathrm{dB}$ change was still found when gaze was away from the attended location. Spatial attention appears to increase speech intelligibility against interferers, with gain decreasing with distance from the focus of attention. Spatial release from masking (RFM) was only found for the attended location. Further experiments suggest that target location uncertainty prevented RFM at unattended locations.
\end{abstract}

Although the cocktail party problem (Cherry, 1953) has been studied for decades, the role of spatial attention in understanding a talker in the presence of competing noise has only recently been explored. Attention may be defined as the allocation of limited neural resources to the processing of a particular target or feature of interest, so that its detection or recognition benefits from enhanced neural processing. Attention can operate in a number of ways. From visual psychophysics, it is known that it can be allocated to particular locations in space, to objects in space, or even to particular features of those objects (for a review, see Desimone \& Duncan, 1995). In audition, attention can be allocated to certain aspects of an auditory stimulus, such as its frequency (e.g., Giard, Fort, Mouchetant-Rostaing, \& Pernier, 2000; Mondor \& Bregman, 1994; Scharf, 1991) or location (e.g., Rhodes, 1987; Teder \& Näätänen, 1994). Allocating auditory attention to a particular location produces increased levels of brain activation (as evidence by evoked potentials), better accuracy, and faster response times (RTs) to nonspeech stimuli when they are at attended locations rather than distant locations (Arbogast \& Kidd, 2000; Mondor \& Zatorre, 1995; Rhodes, 1987; Sach, Hill, \& Bailey, 2000; Teder \& Näätänen, 1994; Teder-Sälejärvi \& Hillyard, 1998; TederSälejärvi, Hillyard, Roder, \& Neville, 1999).

The way in which the processing advantage of spatial attention changes with distance from the focal point of attention is still not well understood. It is a question more easily addressed in vision than in audition, since visual stimuli are imaged directly onto the retina, so that visual space is mapped receptotopically. A number of investi- gations of visual attention have revealed a spatial gradient of attention in vision, with elicited brain responses to stimuli and performance on tasks involving those stimuli, reducing as a function of distance from the spatial focus of attention (Downing, 1988; Mangun \& Hillyard, 1988; Scharlau, 2004).

In audition, stimuli are mapped onto the basilar membrane tonotopically, and spatial location must be computed by comparing information from each ear. Consequently, a spatial focus for attention cannot be assumed a priori. However, previous experiments employing nonspeech sounds have suggested a spatial gradient of auditory attention. Mondor and Zatorre (1995), for example, demonstrated that RTs in a tone-discrimination task became longer as distance from an attended location increased. Teder-Sälejärvi and colleagues have demonstrated lowered detection rates and reduced event-related potentials (ERPs) as distance from an attended location increases (Teder \& Näätänen, 1994; Teder-Sälejärvi \& Hillyard, 1998; Teder-Sälejärvi et al., 1999). These experiments showed a varying spatial gradient, with attentional gradients being steeper at the midline than at lateral positions, and steeper where maskers were closer to the target, constraining the focus of attention (TederSälejärvi et al., 1999).

Evidence of a spatial gradient of attention in the context of speech intelligibility is less clear; there are only a few relevant studies. Kidd, Arbogast, Mason, and Gallun (2005) reported that intelligibility of a speech signal competing with a nontarget speech signal was facilitated when the target was presented at an attended location. Cherry

K. Allen, kachinaa@medsci.usyd.edu.au 
(1953) showed that, in one circumstance, speech at an unattended ear was not processed, but more recent experiments suggest that this may be task dependent. Treisman (1960) demonstrated that speech presented to the unattended ear during dichotic listening can still undergo a degree of informational processing, and Rivenez, Darwin, and Guillaume (2006) showed that speech at an unattended location may be lexically processed under some conditions. To date, no study has demonstrated whether speech intelligibility varies as a function of distance from an attended location. This was investigated as one aim of this study.

Numerous studies have demonstrated that spatially separating a target and masker leads to a release from masking, as measured by improved target discrimination (e.g., Freyman, Balakrishnan, \& Helfer, 2001; Kidd, Mason, Deliwala, Woods, \& Colburn, 1994; Kidd, Mason, Rohtla, \& Deliwala, 1998; Litovsky, 2005; Noble \& Perrett, 2002). Two types of masking have been recognized in this context: energetic masking and informational masking. In energetic masking, the masking effect occurs when components from the nontarget sounds overlap in frequency and time with those of the target signal, causing interference and distortion at the level of the basilar membrane. Informational masking, by contrast, is a more central effect and refers to situations where the target and nontarget signals are confusable at the level of information content and speech comprehension, causing elements of the nontarget signal to become confused with those of the target signal (e.g., Brungart, 2001; Kidd et al., 1994). Spatially separating targets from maskers permits the listener to exploit binaural and spectral cues to source localization and use them to segregate the signals and focus on one sound stream of interest (e.g., Edmonds \& Culling, 2005; Hawley, Litovsky, \& Culling, 2004; Persson, Harder, Arlinger, $\&$ Magnuson, 2001). Indeed, studies using nonoverlapping, frequency-banded speech against speech maskers showed that spatial separation advantages may be greater for informational masking than for energetic (Arbogast, Mason, \& Kidd, 2002).

No previous studies have systematically looked at spatial release from masking at unattended locations. The second aim of these experiments is thus to examine spatial release from masking for target locations beyond the focus of attention.

\section{GENERAL METHOD}

\section{Subjects}

Subjects were seated in a sound-attenuated audiometric booth $(3.5 \times 4.6 \times 2.4 \mathrm{~m})$ lined with $7.5-\mathrm{cm}$-thick acoustic foam to create a semi-anechoic environment. Six Tannoy active loudspeakers were placed $1.3 \mathrm{~m}$ away on the subject's audiovisual horizon at $20^{\circ}$ intervals. Subjects were instructed to remain facing directly ahead at all times, although their heads were not restrained.

For all experiments, subjects had normal hearing, as tested using a standard pure tone audiogram, and spoke English as their first and primary language. No subjects were remunerated for participation, and all gave informed, written consent.

\section{Stimuli}

A speech corpus was recorded, using a single female voice speaking American English. Since broadband signals are important for accurate localization of nonspeech sounds (Carlile, Delaney, \& Corderoy, 1999; Langendijk \& Bronkhorst, 2002) and speech (Best, Carlile, Jin, \& van Schaik, 2005; Jin, Best, Carlile, Baer, \& Moore, $2002)$, the corpus was broadband $(0-22.5 \mathrm{kHz})$, to maximize the acoustic cues to location.

Differences in voice characteristics between talkers are a powerful cue for speech segregation (e.g., Brungart, 2001; Brungart, Simpson, Ericson, \& Scott, 2001; Darwin, Brungart, \& Simpson, 2003; Ericson, Brungart, \& Simpson, 2004). Indeed, when interferers are the same gender as the target, rendering voice characteristic cues less salient, spatial separation of masker and target is more beneficial than when large differences in voice characteristics occur between talkers (e.g., Festen \& Plomp, 1990; Noble \& Perrett, 2002). Using tones, Mondor, Zatorre, and Terrio (1998) showed that location and frequency could not be independently attended to when strong cues were available for each, even though location cues were irrelevant to the experimental task. To minimize voice and spectral cues, and thus maximize the listener's reliance on spatial cues, the same talker was used for both target and maskers, a condition found by Brungart to provide the most difficult of listening conditions.

The corpus used was created using pseudowords, because familiar high-frequency words are easily understood in word recognition tests (Connine, 2004; Luce \& Pisoni, 1998) and might therefore obviate effects of attention. Target words consisted of one of the following pseudowords: "targ," "karg," or "parg." There were two maskers consisting of one pseudoword randomly chosen from the list containing "borg," "dorg," and "gorg," and one from the list containing "boog," "doog," and "goog." To prevent subjects from using any slight differences in recording or articulation between pseudowords as cues, five versions of each pseudoword were recorded and randomly used throughout the experiment. The target word was composed of two components, a single phoneme "p," "t," or "k," followed by the identifying sound "arg." Initial target phonemes were voiceless, whereas maskers utilized the voiced sounds "b," "d," or "g." This ensured that initial energy was greater in the voiced maskers than in the voiceless target, maximizing the difficulty of the task. Selection of masker and target was entirely random, and no attempt was made to mask targets with their phonetically matched voiced pair.

Stimuli were presented using MATLAB (Mathworks, Version 14.1) and a Hammerfall Multiface (RME, Ltd.) sound card at a sampling rate of $44100 \mathrm{~Hz}$ and $57 \mathrm{~dB}$ (SPL). All stimuli were ramped with 10-msec cosine windows to prevent onset or offset clicks, and were standardized for RMS energy.

\section{Procedure}

Target stimuli consisted of one pseudoword randomly selected from the set of "parg," "karg," and "targ." Subjects were instructed to identify the first target phoneme by entering the letter "p," "t," or "k" into a laptop computer. Two pseudoword maskers were played concurrently with the target, one randomly selected from "borg," "dorg," or "gorg," and the other from "boog," "doog," or "goog." When the masker pseudowords were played from locations flanking the target, they were randomly assigned to the left or right side.

Subjects were instructed to attend to a particular location at all times, and the target was played at this location on $80 \%$ of trials. Attentional resources are known to be strategically allocated according to target probability (Sonnadara, Alain, \& Trainor, 2006). Since the specified location had a high target probability, it strongly encouraged subjects to focus their attention on the indicated location as the best strategy for success and most efficient allocation of limited cognitive resources. On the $20 \%$ of trials where the target was at an unexpected location, subjects would not have had time to shift their spatial focus of attention to the new target location, because the deployment of auditory spatial attention is thought to take at least 80-200 msec (Teder-Sälejärvi \& Hillyard, 1998) and the mean duration of the initial "p," "t," or "k" phonemes that distinguish the target word (since all terminate with "arg") is less than $80 \mathrm{msec}$.

The speech reception threshold (SRT) was calculated for each condition and expressed as the level of the target (in $\mathrm{dB}$ ) below the 
level of the combined maskers where $67 \%$ of the initial target phonemes were intelligible (i.e., correctly identified). The SRT threshold was calculated for each subject using a cumulative Gaussian psychometric function fitted to the data using a maximum likelihood procedure (Watson, 1979). Release from masking (RFM) was calculated as

$$
\begin{aligned}
& \text { RFM(separated condition })= \\
& \quad \text { SRT(collocated) - SRT(separated condition). }
\end{aligned}
$$

\section{EXPERIMENT 1A}

\section{Method}

Subjects. Four female (mean age, $30.3 \pm 4.5$ years) volunteers were recruited, including the principal investigator. Three subjects had previously participated in tests of auditory perception.

Procedure. Subjects were instructed to face straight ahead and attend to the loudspeaker located directly in front of them, from which the target would be played in $80 \%$ of trials. They were informed that on $20 \%$ of trials the target would come from one of the other 3 loudspeakers located $20^{\circ}, 40^{\circ}$, or $60^{\circ}$ to one side of the expected loudspeaker (see Figure 1A). In separate blocks, lateral loudspeakers were located either to the left or right side of the attended location. To remove hemispheric bias, all results from blocks using the left and the right hemispheres were later pooled and grouped according to their absolute azimuthal distance from the expected location.

All subjects carried out 200 unrecorded practice trials of collocated and separated conditions (see below), followed by a minimum of 1,000 experimental trials of each condition on both the left and right side. These were broken into blocks of 200. At the beginning of each block, 6 unrecorded practice trials were played from the expected location to ensure that subjects had time to focus attention on this location. Whenever an "unexpected" trial occurred, it was always followed by a trial at the expected (attended) location, so that the subjects had no incentive to shift their focus of attention on subsequent trials. A range of 20-dB signal-to-noise ratios (SNRs) was calculated in each block, with 40 trials being presented at each of five evenly spaced SNR levels. Maximum and minimum SNR levels were varied between blocks depending on the performance of the subject, but all subjects were presented with stimuli between the SNR ranges of -30 and $+10 \mathrm{~dB}$. The majority of trials were presented between -20 and $-0 \mathrm{SNR}$, to provide maximum data around the threshold of interest.

Two conditions were tested: collocated and separated. In the collocated condition, the target and both maskers were played from the same loudspeaker. In the separated condition, the target played from the central loudspeaker, and one masker was played from each of two symmetrically spaced loudspeakers flanking the central loudspeaker at $\pm 20^{\circ}$. Note that for both conditions, the same $80 / 20$ split of expected/unexpected target locations was used. In the collocated condition, the "unexpected" trials simply involved playing the three speech signals (target plus two maskers) from one of the other (unattended) loudspeakers. In the separated condition, a new target location was chosen and the two masker stimuli were shifted accordingly to maintain their flanking positions $20^{\circ}$ to either side of the target.

\section{Results}

Mean SRT data with standard error bars for 4 subjects are shown in Figure 2A for targets located at the attended position of $0^{\circ}$, and also at unexpected locations progressively more distant from the focus of attention $\left(20^{\circ}, 40^{\circ}\right.$, $\left.60^{\circ}\right)$. In the separated condition (solid line), whether at the expected location or not, the target was always flanked symmetrically by maskers at $\pm 20^{\circ}$. These means clearly demonstrate a progressive decrease in performance (increase in SRTs) over the $60^{\circ}$ of distance from the attended location. For the collocated condition (dashed line), there is a change of $2.6 \pm 2.2 \mathrm{~dB} \mathrm{SNR}$, and for the separated condition, there was a change of $5.1 \pm 1.8 \mathrm{~dB}$ SNR. A $2 \times 4$ ANOVA showed a significant linear trend $[F(1,3)=$ $20.82, p<.05]$, with no significant effects of separation $[F(1,3)=10.00, p>.05]$.

From these data we also calculated spatial release from masking (the difference between the collocated and separated conditions from Figure 2A). Figure 3 shows the spatial release from masking data averaged across the four

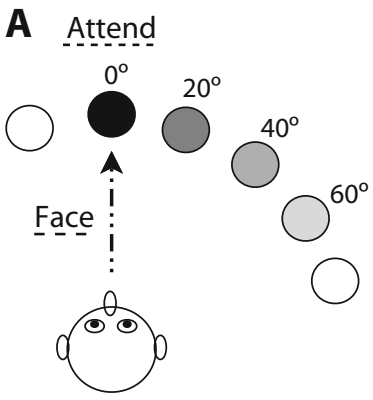

Subject
B

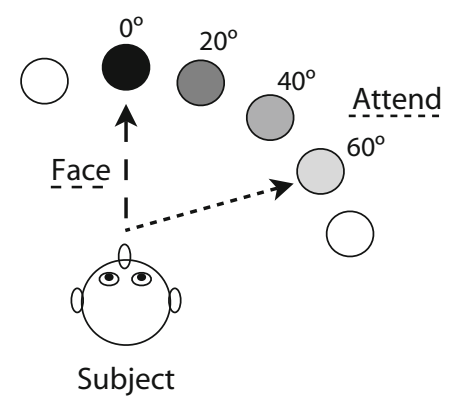

Figure 1. Experimental layouts for Experiment 1. (A) Attend $0^{\circ}$, Face $0^{\circ}$. Subjects faced and attended to the loudspeaker directly ahead from which the target was played on $80 \%$ of trials ("expected" location). (B) Attend $60^{\circ}$, Face $0^{\circ}$. Subjects oriented their head and eyes directly ahead but directed their attention to the loudspeaker located $60^{\circ}$ from the midline from which the target was played on $80 \%$ of trials ("expected" trials). In both layouts, the target in $20 \%$ of trials was played at an unexpected location from a loudspeaker $20^{\circ}, 40^{\circ}$, or $60^{\circ}$ from the expected location. In both layouts, targets were masked by either collocated maskers or by separated maskers $\left( \pm 20^{\circ}\right.$ from the target). Results were collected for layouts on the left-hand and right-hand sides and pooled across hemispheres. 
observers. Spatial unmasking was significant only at the attended location with unattended locations showing no significant unmasking $(z$ tests, $p<.05)$.

Note that, prior to conducting this analysis, we tested whether SRTs differed at the expected location, depending on whether a given trial followed an expected or an unexpected trial. No difference was found. This confirmed the expectation that subjects would deploy their attention on the probable target location and that they did not alter this strategy following occasional trials at unexpected target locations.

\section{EXPERIMENT 1B}

Experiment 1A demonstrated a gradient in speech intelligibility over space as a function of distance from an attended location. However, rather than reflecting a focus of attention at a particular spatial location and dissipation of attention with distance from this location, it might be due to an automatic allocation of attention to the region corresponding to gaze direction. Another possibility is that the gradient of speech intelligibility observed in Experiment $1 \mathrm{~A}$ might simply reflect reduced auditory acuity away from the midline. Experiment 1B aimed to replicate Experiment $1 \mathrm{~A}$ but with gaze direction and attended location dissociated. Subjects attended to a location away from the midline, while maintaining their gaze direction directly ahead.

\section{Method}

Subjects. Four female volunteers were recruited (mean age, 29.3 \pm 6.2 years), including the principal investigator. Three subjects (S1, S2, and S3) had also participated in Experiment 1A.

Procedure. Subjects were instructed to focus their attention on the loudspeaker located $60^{\circ}$ from the midline but to orient their heads and eyes straight ahead $\left(0^{\circ}\right)$. All carried out 200 unrecorded practice trials of collocated and separated conditions, and a minimum of 1,000 experimental trials for each condition, on both the left and right side. The organization of the experiment into blocks, and the SNR levels used, were as in Experiment 1A. Subjects were informed that in $80 \%$ of trials ("expected" trials) the target would be heard from the attended loudspeaker, but that on the remaining $20 \%$ the target would come from one of the other loudspeakers. These loudspeakers were located $20^{\circ}, 40^{\circ}$, or $60^{\circ}$ to the side of the attended loudspeaker (see Figure 1B). In a given block of trials, targets were always presented on either the left or right side, but results were later pooled across hemispheres based on absolute azimuthal distance from the attended location. As in Experiment 1A, there were never two trials in a row from unexpected locations, and we tested both collocated and separated conditions (where maskers were played from two flanking loudspeakers located $\pm 20^{\circ}$ from the loudspeaker playing the target).

\section{Results}

The SRT data averaged across 4 subjects are shown in Figure 2B for both the collocated (dashed line) and separated (solid line) conditions. As in Experiment 1A, in the separated condition, the target was always flanked symmetrically by maskers at $\pm 20^{\circ}$ whether at an attended or unattended position. Eighty percent of targets were played from the attended position $60^{\circ}$ from the midline, with $6.6 \%$ of targets at each of the unattended locations located at $0^{\circ}, 20^{\circ}, 40^{\circ}$, and $60^{\circ}$ from the subject's gaze. Thus, greater distance from the midline corresponds to locations

\section{Mean Speech Reception Thresholds}
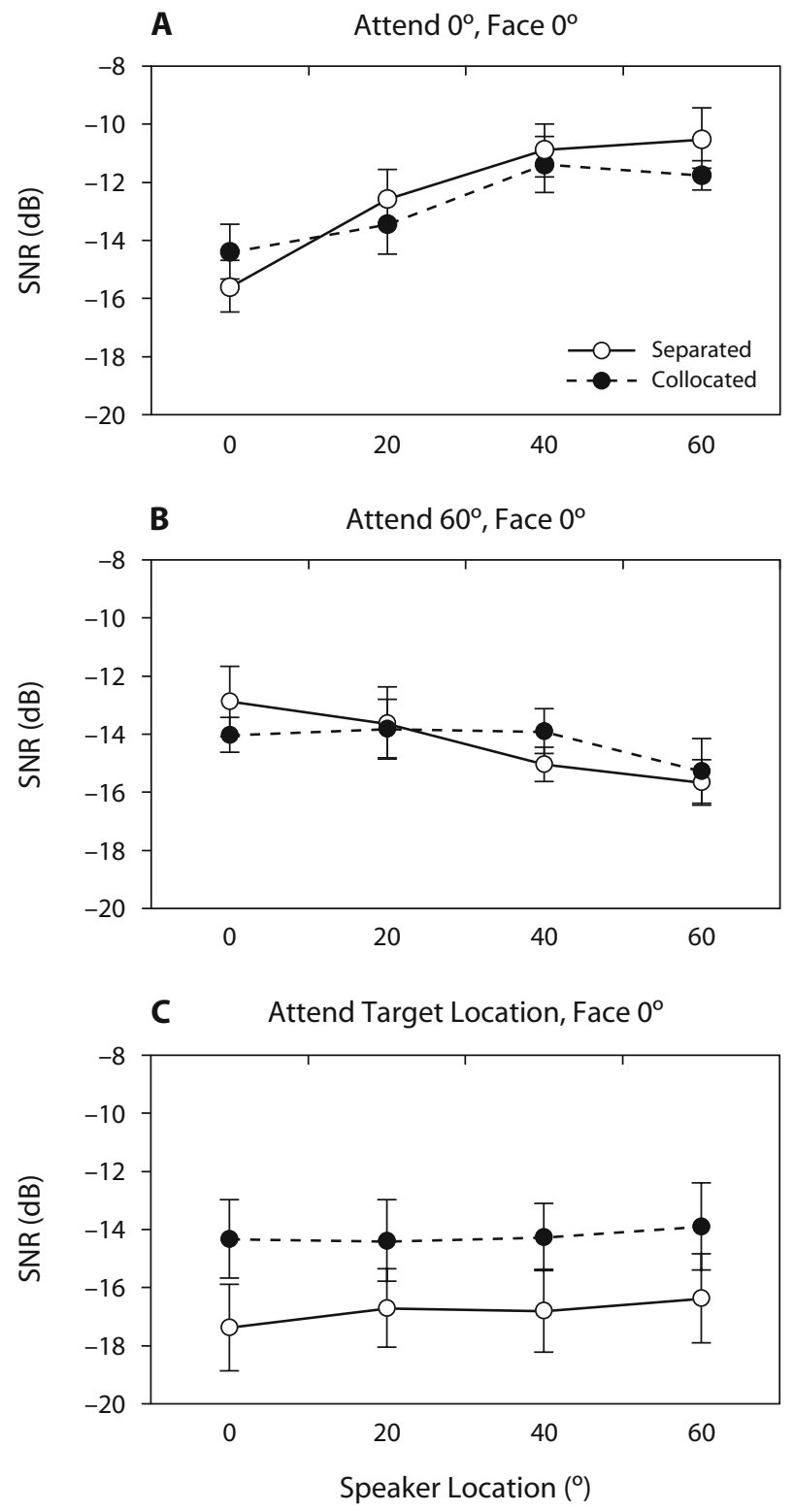

Figure 2. Data from Experiment 1. Speech reception thresholds (SRTs) averaged over the 4 listeners with \pm 1 standard error bars. Maskers were either collocated with the target or symmetrically separated by $\pm 20^{\circ}$ (see the Method sections). Head and eyes were oriented straight ahead at $0^{\circ}$ for all conditions. (A) Subjects attended to the speaker directly in front $\left(0^{\circ}\right)$ which played $80 \%$ of trials, with $20 \%$ randomly played from one of the other speakers. Subjects show steadily increasing SRTs (decreasing performances) with distance from the attended speaker. (B) Subjects attended to the speaker at $60^{\circ}$ which played $80 \%$ of trials, with the remaining $20 \%$ played randomly from another speaker. Subjects showed steadily decreasing SRTs (increasing performances) with reduced distance from the attended speaker. (C) Subjects were told from which speaker all trials in a block would be played and attended to it. Subjects showed no effect of speaker location on performance. SNR, signal-to-noise ratio.

closer to the focus of attention. The results were similar to those from Experiment 1A. For the separated condition data demonstrate progressively increasing perfor- 


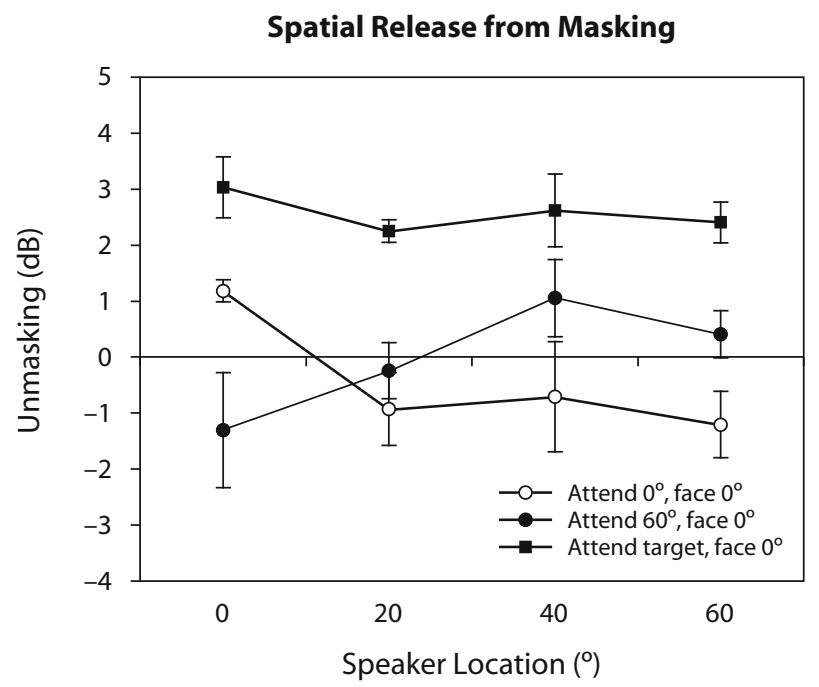

Figure 3. Data from Experiment 1, showing spatial release from masking. The data were calculated as the SRT difference between collocated and separated conditions, averaged over the 4 observers. The conditions were the same as described in Figure 2. Significant unmasking occurs for all speakers in the "attend to target" condition and for the attended speaker in the "face 0 " condition. Error bars show \pm 1 standard error.

mance (decreasing SRTs) with proximity to the attended location. In this condition, there was an overall change of $2.9 \pm 0.9 \mathrm{~dB}$ SNR over the $60^{\circ}$ of azimuthal distance. A $2 \times 4$ ANOVA showed a significant linear trend $[F(1,3)=$ $41.09, p<.01]$ with no significant interaction of level of separation $[F(1,3)=0.019, p=.9]$.

From these data, we also calculated spatial release from masking (the difference between the collocated and separated conditions from Figure 2B) across the 4 observers at the attended and unattended locations. This is shown in Figure 3. Spatial unmasking was not significant at any of the azimuthal locations $(z$ tests, $p<.05)$.

\section{EXPERIMENT 1C}

In Experiment 1A, spatial release from masking was significant only for the attended loudspeaker located straight ahead of the subject (Figure 3). In Experiment 1B, spatial release from masking was not significant for the attended loudspeaker (located $60^{\circ}$ laterally), nor for any of the other loudspeaker locations closer to the midline (Figure 3). This failure to find spatial release from masking may have been due to the direction-dependent filtering imposed by the outer ear (Carlile \& Pralong, 1994). Peak gains in the $2-4 \mathrm{kHz}$ range of up to $15 \mathrm{~dB}$ have been found to occur for horizontal angles between $30^{\circ}$ and $60^{\circ}$ from the midline. Because Experiments 1A and 1B utilized loudspeaker locations within the $0^{\circ}-60^{\circ}$ range, it is possible that the SNR in the separated condition would depend in part on the location of the target and masker signals within this range. Thus, the lack of spatial release from masking at lateral positions found in Experiment 1B might be accounted for in terms of acoustic differences in SNR between the collocated and separated conditions. To test this possibility, each loudspeaker used in Experiments $1 \mathrm{~A}$ and $1 \mathrm{~B}$ was tested for spatial release from masking at that location.

\section{Method}

Subjects. One male and 3 female volunteers were recruited (mean age, $28.3 \pm 4.9$ years), including the principal investigator. Two subjects (S2 and S3) had participated in both Experiments 1 and 2, and S4 had completed Experiment 1B. All subjects had previously participated in tests of auditory perception.

Procedure. Subjects were instructed to face straight ahead at all times and to attend to the location of the loudspeaker indicated. For each loudspeaker, one block of 150 trials was completed for both the collocated and the separated conditions. This included 30 trials at each of the following SNR levels: $-30,-22.5,-7.5$, and $0 \mathrm{~dB}$. The results for the first 6 trials for each block were not recorded. As before, blocks were repeated on both sides and the results pooled across hemispheres. In the separated condition, the target was always flanked by two symmetrical maskers $\pm 20^{\circ}$ from the target.

\section{Results}

SRTs were calculated for the collocated and separated conditions at all loudspeaker locations. No significant differences between any of the locations were found (Figure 2C; Friedman's test, $p>.05$ ). This indicates that the changes in SRT as a function of distance from the midline in Experiment 1A cannot be explained by variation in acoustic factors across azimuthal location. Similarly, the differences in SRT results between Experiments 1A and $1 \mathrm{~B}$ could not have resulted from the difference between gaze direction and attended auditory location. This supports the conclusion that it is the distance from the attended location that creates the gradient in speech intelligibility in both Experiments 1A and 1B.

Significant spatial release from masking, the difference between collocated and separated conditions, was found at all locations, with no significant differences between locations (Figure 3; Friedman's test, $\alpha=.05$ ). The lack of spatial release from masking at unattended locations in Experiment 1A was, therefore, not a result of location-dependent acoustic differences. Moreover, the fact that significant spatial release from masking was demonstrated at all locations in Experiment 1C means that the failure to find spatial release from masking in Experiment $1 \mathrm{~B}$ at any location similarly cannot be attributed to acoustic factors.

\section{Discussion}

In both Experiments 1A and 1B, subjects performed better at the attended location than at any unattended location (Figures 2A and 2B). This is consistent with previous work showing that attention to a spatial location improves speech processing at that location (Kidd, Arbogast, et al., 2005). More specifically, SRTs increased systematically with distance from the attended location, suggesting a gradient of intelligibility such that speech is better processed closer to the focus of spatial attention (Figures 2A and $2 \mathrm{~B}$ ). These results corroborate previous findings with nonspeech stimuli suggesting a spatial gradient of attention in audition. For example, as distance from an attended 
location increases, RTs to tone stimuli become slower and the corresponding ERP responses are reduced (Mondor \& Zatorre, 1995; Teder \& Näätänen, 1994; Teder-Sälejärvi \& Hillyard, 1998; Teder-Sälejärvi et al., 1999). Our results support the theory of a spatial gradient of attention in audition and extend the role of attention to the speech domain.

An alternative interpretation for the gradient of speech intelligibility in Experiment 1A would be that it simply reflects the localization acuity of the auditory system. Numerous studies have shown that stimuli at lateralized positions are not as well processed as are those at positions nearer to the midline (e.g., Carlile, Leong, \& Hyams, 1997; Mills, 1958). Furthermore, Lie and Branch Coslett (2006) and Rorden and Driver (1999) demonstrated that gaze direction provides a processing advantage, possibly due to an increase in the allocation of attention to the region corresponding to gaze direction. Thus, the apparent gradient of attention could also have been due to observers' gazes being directed forward toward the attended central loudspeaker. Experiment 1B was designed to test whether the intelligibility gradient demonstrated in Experiment $1 \mathrm{~A}$ is due to an effect of gaze direction, or acuity, or whether it was a genuine gradient of attention that falls off with distance from an attended spatial location. Overall, consistent with Experiment 1A, Experiment 1B again showed an intelligibility gradient peaking at the spatial focus of attention. This gradient is evident despite a $60^{\circ}$ dissociation between gaze direction and the spatial focus of attention, and an increase in auditory spatial acuity toward the midline, both of which would have predicted the opposite result. The results of Experiment 1C (Figure 2C) demonstrate that subjects performed equally well in both the collocated and separated conditions at all loudspeaker locations. Thus, neither gaze direction nor spatial acuity was driving the gradient of speech intelligibility in Experiments $1 \mathrm{~A}$ and $1 \mathrm{~B}$.

Experiments $1 \mathrm{~A}$ and $1 \mathrm{~B}$ show no spatial release from masking at unattended positions, but the results from Experiment 1C showed equivalent spatial release from masking at all speaker locations. It differs from Experiments $1 \mathrm{~A}$ and $1 \mathrm{~B}$ in that the stimuli in Experiment $1 \mathrm{C}$ were presented with $100 \%$ certainty at the attended location, as opposed to only a $6.6 \%$ likelihood in unattended speakers in Experiments $1 \mathrm{~A}$ and 1B. Sonnadara et al. (2006) demonstrated that the allocation of attentional resources is linked to target location probability; the relative increase in attentional resources in Experiment $1 \mathrm{C}$, therefore, could explain the release from masking seen in each of the locations.

\section{EXPERIMENT 2}

In Experiments $1 \mathrm{~A}$ and $1 \mathrm{~B}$, we observed little or no spatial release from masking at unattended locations (see Figure 3). One potential explanation for this is given on the basis of target location uncertainty. In the collocated condition, there is little uncertainty about the target location, since there is only one single loudspeaker playing the sound. In the separated condition there are three loudspeakers playing at once, which increases target lo- cation uncertainty. Although subjects are aware of which loudspeaker will play the target during an expected trial, during an unexpected (separated) trial, three loudspeakers sound and there will be more uncertainty about which plays the target location. Kidd, Arbogast, et al. (2005) and Shinn-Cunningham and Ihlefeld (2004) have demonstrated reduced performance in trials where the target location was uncertain. Thus, the lack of spatial release from masking in the unexpected trials could potentially be explained by reduced target performance in the separated condition due to location uncertainty counteracting any benefits arising from spatial separation of the maskers. Experiment 2 aimed to explore this possibility.

\section{Method}

Subjects. Four female volunteers (mean age, $32 \pm 2.7$ years) were recruited, including the principal investigator. Two subjects (S3, S4) had also participated in Experiments 1A, 1B, and 1C. Subjects S1 and S2 had previously undertaken other auditory psychophysical experiments.

Procedure. Subjects sat facing directly ahead at the central loudspeaker of an array of three equidistant, equally spaced loudspeakers, $20^{\circ}$ apart in azimuth. Subjects were instructed to face straight ahead at all times and to attend to the location of the loudspeaker indicated. Trials were carried out in blocks of 100 for conditions 1 and 2 and in blocks of 200 for conditions 3 to 6 (conditions described below). Each block consisted of trials at five evenly spaced SNR levels spanning $20 \mathrm{~dB}$. All conditions were tested at evenly spaced SNR levels between -30 and $0 \mathrm{~dB}$, with most trials being gathered in the range -20 to $-10 \mathrm{~dB}$ SNR to maximize data around the threshold of interest. The first six trials of each block were for practice and were not recorded. The six conditions were the following.

Collocated, one possible location. Subjects faced directly ahead and attended to one of the three loudspeakers as indicated. Two maskers and a target were played from that loudspeaker.

Separated, one possible location. Subjects faced directly ahead and attended to one of the three loudspeakers as indicated. A target was played from the indicated loudspeaker and a single masker was played from each of the other loudspeakers.

Collocated, two possible locations. Subjects faced directly ahead and were told the target location would be equally distributed between the loudspeaker on the left and the central loudspeaker. A target and two maskers were played from the randomly chosen loudspeaker (left or central).

Separated, two possible locations. Subjects faced directly ahead and were told the target location would be equally distributed between the loudspeaker on the left and the central loudspeaker. A target was played randomly from one of the two indicated loudspeakers and a single masker was played from each of the other two loudspeakers.

Collocated, three possible locations. Subjects faced directly ahead and were instructed that the target could be played from any of the three loudspeakers with equal probability. A target and two maskers were played from the randomly chosen loudspeaker (left, central, or right).

Separated, three possible locations. Subjects faced directly ahead and were instructed that the target could be played from any of the three loudspeakers with equal probability. A target was played from the randomly chosen loudspeaker and a single masker was played from each of the other two loudspeakers.

\section{Results and Discussion}

Figure 4 shows mean SRTs obtained from the 4 subjects for the collocated (dashed line) and separated (target flanked by maskers at $\pm 20^{\circ}$, solid line) conditions. They are presented when the target was known to be at the cen- 
tral loudspeaker (one possible target location), when the target was played from one of two equally likely possible locations, and when the target was played from one of three equally likely possible locations.

After the cumulative Gaussian was fitted to the data (see the General Method section), a bootstrapping procedure (Efron \& Tibshirani, 1994) was used to sample each psychometric function 1,000 times. The standard deviation of the distribution of thresholds obtained from the 1,000 resampled functions was used to enable post hoc $t$ tests $(\alpha=.05)$ to be carried out on thresholds between conditions (see Alais \& Carlile, 2005).

Post hoc $t$ tests $(p>.05)$ with Bonferroni corrections showed that SRTs were not significantly different between the three collocated conditions. Calculating spatial release from masking by comparing the SRTs for the collocated and separated conditions demonstrates differences in levels of unmasking between the conditions (ANOVA) $[F(2,9)=$ $13.77, p<.002]$. This unmasking was significant when the location of the target was known and when the target had two possible locations (Figure 5) $(z$ test, $p<.05)$. However, when the target had three possible locations, there was no significant unmasking ( $z$ test, $p>.05$ ).

Where the target location was known and could therefore be attended, spatial unmasking is clearly present. When there was a choice of three possible target locations, so that allocation of attention was much more difficult, no spatial release from masking was found (Figure 5). Because SRTs for collocated trials are not significantly different between conditions (Figure 4), it was therefore the SRTs for the separated condition which decreased in each case. To ensure that subjects knew that the target could only come from one of three locations, only three loudspeakers were used in this experiment. This meant that in the separated conditions, the central loudspeaker was flanked by two symmetrical maskers, whereas, for example, the leftmost loudspeaker had two maskers only to the right. The best approximation to the separated conditions in Experiments $1 \mathrm{~A}, 1 \mathrm{~B}$, and $1 \mathrm{C}$ was when the target was played from the central loudspeaker, since in all cases the target was always symmetrically flanked by two maskers at $\pm 20^{\circ}$. Therefore, data discussed in this article, and presented in Figures 4 and 5, only include the results from trials where the target was played from this central speaker.

The observation that unmasking was absent when the target had three possible locations supports the hypothesis that it was uncertainty about target location in the separated conditions that reduced any advantage afforded by spatial release from masking when target and maskers were separated.

\section{GENERAL DISCUSSION}

Overall, the results from these experiments support three conclusions: (1) Auditory attention can be spatially allocated to a known target location to improve auditory perception at the attended location, (2) there is a gradient of attention such that the effect of attention falls off with distance from its spatial focus, and (3) spatial release from masking relies in part on a prior knowledge of the target location.

Experiments 1A and 1B are consistent with results from Kidd, Arbogast, et al. (2005), which showed advantages in speech intelligibility in multitalker situations at an attended location. Certainly in both Experiments 1A and $1 \mathrm{~B}$, SRTs were significantly lower (better performance) at the attended location than at an unattended location, $60^{\circ}$ from the focus of attention. Experiment $1 \mathrm{~A}$ also demonstrated a gradient of speech intelligibility as a function of distance from the attended location. This was confirmed in Experiment 1B, because this gradient was still present when the attended location was $60^{\circ}$ from the saggital midline. Furthermore, performances for unattended locations nearer the midline were worse than they were at the focus of attention, even though better resolution closer to the midline would have predicted the opposite. These results are consistent with previous studies using tone stimuli showing that performance declined and ERP components reduced with distance from an attended location (Mondor \& Zatorre, 1995; Teder \& Näätänen, 1994; Teder-Sälejärvi \& Hillyard, 1998; TederSälejärvi et al., 1999), these results extend those findings to speech intelligibility.

Experiment $1 \mathrm{~B}$ tested whether the gradient of intelligibility found in Experiment 1A may have simply been due to reduced auditory acuity away from the midline. This was done by having subjects attend $60^{\circ}$ from the midline, and by measuring intelligibility for locations back toward the midline. Also, by having a subject's head and eyes oriented directly ahead, we dissociated gaze direction from the di-

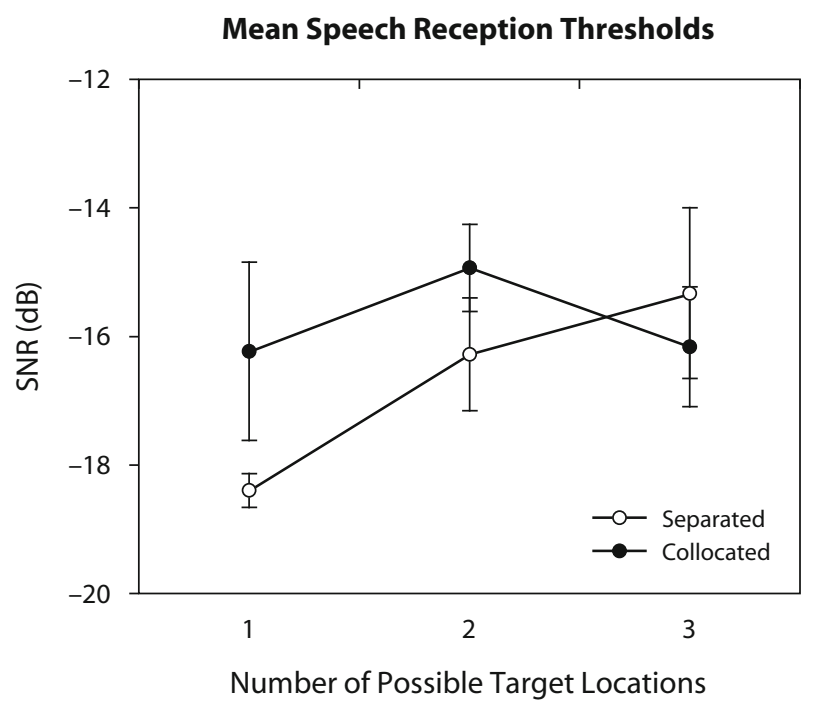

Figure 4. Data from Experiment 1B showing SRTs (67\% correct) from the central loudspeaker, averaged over the 4 subjects. The target was played from a single known location, from one of two equally possible locations, or from one of three equally likely locations. Error bars show \pm 1 standard error. In collocated conditions, no significant differences were found between the three levels of positional uncertainty. In the separated conditions, significant differences between a known target location and a possible choice of three were found. SNR, signal-to-noise ratio. 
Spatial Release From Masking

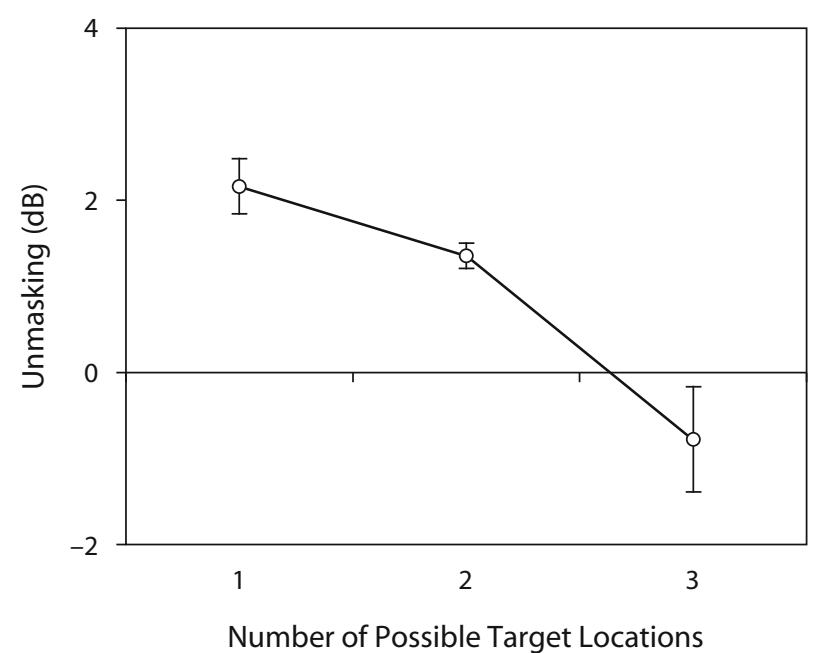

Figure 5. Data from Experiment 1B, showing spatial release from masking for the central speaker. The data show the SRT difference between the collocated and separated conditions for three levels of positional uncertainty: one possible target location, two possible target locations, and three possible target locations. Error bars show \pm 1 standard error. Significant unmasking ( $z$ test, $a=.05$ ) is found for the single possible location and for two possible locations.

rection of conscious attention. The importance of Experiment $1 \mathrm{~B}$ is twofold. First, it demonstrates that the intelligibility gradient found in Experiment 1A was not simply a reflection of reduced spatial acuity away from the midline. Second, the gradient was demonstrated while the directions of gaze and attention were dissociated. This confirms that the intelligibility gradient found in Experiment 1A was not simply due to a preference to allocate attentional resources in the direction of gaze (Lie \& Branch Coslett, 2006).

One other possible explanation for a gradient of intelligibility could be that it is due to the time taken to switch attention between the expected and unexpected locations. If the time required to switch attention to an unexpected location was proportional to distance, a gradient in performance would also be expected. From the attentional shift times in Rhodes's (1987) experiments, attentional shifts require about $2.8 \mathrm{msec} / \mathrm{deg}$. Thus, shifts of $20^{\circ}$ to $60^{\circ}$ would take between 56 and $168 \mathrm{msec}$. Using ERPs, Teder-Sälejärvi and Hillyard (1998) estimated that an initial shift of attention to a new location takes about 80 to $200 \mathrm{msec}$. Because the first phoneme in the corpus used in the present study (the key to correct identification) was less than $80 \mathrm{msec}$, attentional switching would not have been possible. This indicates that fast switching of attention between locations is an unlikely explanation of the results reported here.

In Experiments 1A and 1B, spatial release from masking was not found at unattended locations; however, Experiment $1 \mathrm{C}$ demonstrated that spatial release from masking was found at all locations when the target location was known in advance. Experiment 2 showed that uncertainty about target location negated the performance advantages of spatially separating target and maskers. This is consistent with previous results (Kidd, Arbogast, et al., 2005) showing that identification performance for a known target $(92 \%)$ was dramatically reduced when the target had three possible locations (67\%). Similarly, a significant degradation of performance was shown by Shinn-Cunningham and Ihlefeld (2004) when a known target location was compared with one randomized between two locations. However, in that study, spatial release from masking was not found to be affected by randomization. This may be attributable to their using the coordinate response measure as stimulus. This stimulus consists of a carrier sentence of the form "Ready 'call-sign' go to 'color' 'number' now," where target locations are identified by the call sign, and the color and number are the scoring words. A potential problem arises due to the length of these sentences. Once the call sign has been identified, there is time to reallocate the focus of attention to the target location (a process which takes as little as $80 \mathrm{msec}$; Teder-Sälejärvi \& Hillyard, 1998) while the words "go to" are spoken, and before the scoring words. By comparison, in Experiments 1 and 2, we used very brief stimuli- the first phoneme of a target pseudoword-so that subjects could not have identified and switched their attention to the target location prior to the scoring phoneme. This would preserve considerable uncertainty about target location, countering advantages from spatial release from masking.

Finally, the spatial release from masking reported in Experiment $1 \mathrm{~A}$ at the attended location was relatively small $(1.2 \mathrm{~dB})$, compared with $2.2 \mathrm{~dB}$ for the central loudspeaker in the location-known condition of Experiment 2. Both values are also lower than the 4.5-dB unmasking found by Noble and Perrett (2002) for a symmetrical spatial separation of $\pm 30^{\circ}$ with a female target and two maskers. Same-gender target and maskers tend to produce high levels of information masking (Brungart, 2001) and consequently larger spatial release from masking (e.g., Brungart, 2001; Brungart et al., 2001; Festen \& Plomp, 1990; Freyman et al., 2001; Hall, Buss, \& Grose, 2005; Kidd, Mason, \& Gallun, 2005; Kidd et al., 1998; Noble \& Perrett, 2002; Rhebergen, Versfeld, \& Dreschler, 2005). Using the same talker for target and maskers in this study maximized informational masking while maintaining considerable energetic masking because of identical voice characteristics. This high level of energetic masking, and the narrower spatial separation of $20^{\circ}$, were most likely partly responsible for the relatively low level of unmasking reported here.

\section{Conclusion}

Speech intelligibility was facilitated at an attended location and declined as a function of distance from the attended location. This was true whether the attended location was on the midline or lateralized by $60^{\circ}$. This facilitation was not caused by gaze direction and the decline cannot be due to rapid shifting of the focus of attention. At unattended locations, there was no spatial release from masking. This likely resulted from uncertainty about target 
location when target and maskers were separated, which countered any benefits of spatial separation.

\section{AUTHOR NOTE}

We thank Karen Froud and Deborah Apthorp for their assistance with the work and Teachers College, Columbia University, for the generous use of equipment and resources. This work was supported by the Australian Research Council. Correspondence concerning this article should be addressed to K. Allen, Department of Physiology, University of Sydney, Sydney, NSW 2106, Australia (e-mail: kachinaa@medsci.usyd.edu.au).

Note-Accepted by the previous editorial team, when Thomas H. Carr was Editor.

\section{REFERENCES}

Alais, D., \& Carlile, S. (2005). Synchronizing to real events: Subjective audiovisual alignment scales with perceived auditory depth and speed of sound. Proceedings of the National Academy of Sciences, 102, 2244-2267.

Arbogast, T. L., \& KidD, G., JR. (2000). Evidence for spatial tuning in informational masking using the probe-signal method. Journal of the Acoustical Society of America, 108, 1803-1810.

Arbogast, T. L., Mason, C. R., \& Kidd, G., JR. (2002). The effect of spatial separation on informational and energetic masking of speech. Journal of the Acoustical Society of America, 112, 2086-2098.

Best, V., Carlile, S., Jin, C., \& van Schaik, A. (2005). The role of high frequencies in speech localization. Journal of the Acoustical Society of America, 118, 353-363.

BRUNGART, D. S. (2001). Informational and energetic masking effects in the perception of two simultaneous talkers. Journal of the Acoustical Society of America, 109, 1101-1109.

Brungart, D. S., Simpson, B. D., Ericson, M. A., \& Scott, K. R. (2001). Informational and energetic masking effects in the perception of multiple simultaneous talkers. Journal of the Acoustical Society of America, 110, 2527-2538.

Carlile, S., Delaney, S., \& Corderoy, A. (1999). The localisation of spectrally restricted sounds by human listeners. Hearing Research, 128, 175-189.

Carlile, S., Leong, P., \& Hyams, S. (1997). The nature and distribution of errors in the localization of sounds by humans. Hearing Research, 114, 179-196.

Carlile, S., \& Pralong, D. (1994). The location-dependent nature of perceptually salient features of the human head-related transfer functions. Journal of the Acoustical Society of America, 95, 3445-3459.

Cherry, E. C. (1953). Some experiments on the recognition of speech, with one and with two ears. Journal of the Acoustical Society of America, 25, 975-979.

ConNine, C. M. (2004). It's not what you hear but how often you hear it: On the neglected role of phonological variant frequency in auditory word recognition. Psychonomic Bulletin \& Review, 11, 1084-1089.

Darwin, C. J., Brungart, D. S., \& Simpson, B. D. (2003). Effects of fundamental frequency and vocal-tract length changes on attention to one of two simultaneous talkers. Journal of the Acoustical Society of America, 114, 2913-2922.

Desimone, R., \& Duncan, J. (1995). Neural mechanisms of selective visual attention. Annual Review of Neuroscience, 18, 193-222.

DownING, C. J. (1988). Expectancy and visual-spatial attention: Effects on perceptual quality. Journal of Experimental Psychology: Human Perception \& Performance, 14, 188-202.

Edmonds, B. A., \& Culling, J. F. (2005). The spatial unmasking of speech: Evidence for within-channel processing of interaural time delay. Journal of the Acoustical Society of America, 117, 3069-3078.

Efron, B., \& Tibshirani, R. J. (1994). An introduction to the bootstrap. New York: Chapman \& Hall.

Ericson, M. A., BRUNGART, D. S., \& Simpson, B. D. (2004). Factors that influence intelligibility in multitalker speech displays. International Journal of Aviation Psychology, 14, 313-334.

Festen, J. M., \& Plomp, R. (1990). Effects of fluctuating noise and interfering speech on the speech-reception threshold for impaired and normal hearing. Journal of the Acoustical Society of America, 88, 1725-1736.
Freyman, R., Balakrishnan, U., \& Helfer, K. (2001). Spatial release from informational masking in speech recognition. Journal of the Acoustical Society of America, 109, 2112-2122.

Giard, M.-H., Fort, A., Mouchetant-Rostaing, Y., \& Pernier, J. (2000). Neurophysiological mechanisms of auditory selective attention in humans. Frontiers in Bioscience, 5, D84-D94.

HaLl, J. W., III, Buss, E., \& Grose, J. H. (2005). Informational masking release in children and adults. Journal of the Acoustical Society of America, 118, 1605-1613.

Hawley, M., Litovsky, R., \& Culling, J. (2004). The benefit of binaural hearing in a cocktail party: Effect of location and type of interferer. Journal of the Acoustical Society of America, 115, 833-843.

Jin, C., Best, V., Carlile, S., Baer, T., \& Moore, B. (2002). Speech localization. In Proceedings of the Audio Engineering Society, 112th Convention. Munich, Germany.

Kidd, G., JR., Arbogast, T. L., Mason, C. R., \& Gallun, F. J. (2005). The advantage of knowing where to listen. Journal of the Acoustical Society of America, 118, 3804-3815.

Kidd, G., JR., Mason, C. R., Deliwala, P. S., Woods, W. S., \& ColBURN, H. S. (1994). Reducing informational masking by sound segregation. Journal of the Acoustical Society of America, 95, 3475-3480.

Kidd, G., JR., Mason, C. R., \& Gallun, F. J. (2005). Combining energetic and informational masking for speech identification. Journal of the Acoustical Society of America, 118, 982-992.

Kidd, G., JR., Mason, C. R., Rohtla, T. L., \& Deliwala, P. S. (1998). Release from masking due to spatial separation of sources in the identification of nonspeech auditory patterns. Journal of the Acoustical Society of America, 104, 422-431.

LangendiJK, E. H., \& Bronkhorst, A. W. (2002). Contribution of spectral cues to human sound localization. Journal of the Acoustical Society of America, 112, 1583-1596.

Lie, E., \& BRANCH CosLett, H. (2006). The effect of gaze direction on sound localization in brain-injured and normal adults. Experimental Brain Research, 168, 322-336.

LitovsKY, R. Y. (2005). Speech intelligibility and spatial release from masking in young children. Journal of the Acoustical Society of America, 117, 3091-3099.

Luce, P. A., \& Pisoni, D. B. (1998). Recognizing spoken words: The neighborhood activation model. Ear \& Hearing, 19, 1-36.

Mangun, G. R., \& Hillyard, S. A. (1988). Spatial gradients of visual attention: Behavioral and electrophysiological evidence. Electroencephalography \& Clinical Neurophysiology, 70, 417-428.

Mills, A. W. (1958). On the minimum audible angle. Journal of the Acoustical Society of America, 30, 237-246.

Mondor, T. A., \& Bregman, A. S. (1994). Allocating attention to frequency regions. Perception \& Psychophysics, 56, 268-276.

Mondor, T. A., \& Zatorre, R. J. (1995). Shifting and focusing auditory spatial attention. Journal of Experimental Psychology: Human Perception \& Performance, 21, 387-409.

Mondor, T. A., Zatorre, R. J., \& Terrio, N. A. (1998). Constraints on the selection of auditory information. Journal of Experimental Psychology: Human Perception \& Performance, 24, 66-79.

Noble, W., \& Perrett, S. (2002). Hearing speech against spatially separate competing speech versus competing noise. Perception \& Psychophysics, 64, 1325-1336.

Persson, P., Harder, H., Arlinger, S., \& Magnuson, B. (2001). Speech recognition in background noise: Monaural versus binaural listening conditions in normal-hearing patients. Otology \& Neurotology, 22, 625-630.

Rhebergen, K. S., Versfeld, N. J., \& Dreschler, W. A. (2005). Release from informational masking by time reversal of native and non-native interfering speech. Journal of the Acoustical Society of America, 118, 1274-1277.

RHODEs, G. (1987). Auditory attention and the representation of spatial information. Perception \& Psychophysics, 42, 1-14.

Rivenez, M., Darwin, C. J., \& Guillaume, A. (2006). Processing unattended speech. Journal of the Acoustical Society of America, 119, 4027-4040.

Rorden, C., \& Driver, J. (1999). Does auditory attention shift in the direction of an upcoming saccade? Neuropsychologia, 37, 357-377.

SaCh, A., Hill, N., \& Bailey, P. (2000). Auditory spatial attention using interaural time differences. Journal of Experimental Psychology: Human Perception \& Performance, 26, 717-729. 
SCHARF, B. (1991). Frequency selectivity, critical bands, and focused attention. Journal of the Acoustical Society of America, 91, 2356.

SCHARLAU, I. (2004). The spatial distribution of attention in perceptual latency priming. Quarterly Journal of Experimental Psychology, 57A, 1411-1436.

Shinn-Cunningham, B., \& Ihlefeld, A. (2004). Selective and divided attention: Extracting information from simultaneous sound sources. In Proceedings of the 10th ICAD 04 Meeting of the International Conference on Auditory Display. Sydney, Australia.

Sonnadara, R. R., Alain, C., \& Trainor, L. J. (2006). Effects of spatial separation and stimulus probability on the event-related potentials elicited by occasional changes in sound location. Brain Research, 1071, 175-185.

TEDER, W., \& NÄÄTÄNEN, R. (1994). Event-related potentials demonstrate a narrow focus of auditory spatial attention. NeuroReport, 5, 709-711.
Teder-Sälejärvi, W., \& Hillyard, S. (1998). The gradient of spatial auditory attention in free field: An event-related potential study. Perception \& Psychophysics, 60, 1228-1242.

Teder-Sälejärvi, W., Hillyard, S., Roder, B., \& Neville, H. (1999) Spatial attention to central and peripheral auditory stimuli as indexed by event-related potentials. Cognitive Brain Research, 8, 213-227.

Treisman, A. (1960). Contextual cues in selective listening. Quarterly Journal of Experimental Psychology, 12, 242-248.

Watson, A. B. (1979). Probability summation over time. Vision Research, 19, 515-522.

(Manuscript received June 21, 2007; revision accepted for publication July 21, 2008.) 